\title{
Secure Data Transmission Through Zig-bee Module
}

\author{
Suryansh Singh Thakral ${ }^{1}$, Preet Jain ${ }^{2}$ \\ ${ }^{1}$ Pursuing M.E Embedded \& VLSI, Department of ECE, SVITS College Indore, India \\ ${ }^{2}$ Head of Department, Department of ECE, SVITS College Indore, India
}

\begin{abstract}
Wireless technology has important role in today's world every vehicle operates hard functions in a wire connection. Using wireless connection in place of wired connection could prove to be productive in economic senses as it will have an effect on the weight, cost and performance. This process is useful in modern day to day applications of wireless communication. This paper proposes the design of secure data transmission; Enter the message this message is encrypted and then it is transmitted by Zig-bee module. The data which is entered at the transmitter section will be displayed on the GLCD for our convenience and so that data entry can be done in easier way. At the receivers end when the encrypted message is decrypted and is displayed on the GLCD. The mode of communication that used in this project is duplex data transmission over radio frequency. In this paper we propose a fast and secure encryption decryption technique using through which we can encode data and image
\end{abstract}

Keywords: ARM Cortex-M4, Zig-bee module, Cryptography Techniques

\section{Introduction}

From the last few years we have witnessed a vast expansion in wireless communication. Wireless communication is not as secured and reliable. Many algorithms and technologies have been used till now to overcome this problem. This project basically deals with the transmission of data in a secure manner. For this purpose Zig-bee module is used for wireless communication. XBEE and XBEEPRO are the RF modules which are used as Zig-bee devices in the transmission and receiver section. The major area in this project is to encrypt the message according to a specific algorithm on the transmitter side and then send this message using ZIG-BEE transmitter. On the receiver side the message is decrypted and is displayed on the LCD.

Zig-bee module is used in this project which is:

1) Simpler and less expensive

2) Data rate is $250 \mathrm{kbps}$

3) Low power consumption, a battery powered Zig-bee module last up to 10 years

4) Long Distance Transmission, up to 1.5 K.M in open air.

For security purpose an algorithm is implemented in order to encrypt and decrypt the data. Message is encrypted and then sent through wireless module at the receiver section message is then decrypted after entering the provided password. Therefore secure message transmission can take place

\section{Introduction to Cryptography}

Cryptography is a process in which a plain message is converted to cipher text message which is based on an algorithm that both sender and receiver is familiar with, so therefore the cipher text message can be obtained to its original form. So that, a message cannot be read by anyone except the authorized receiver. The process of converting a plain message to its cipher form is called enciphering. Reversing this process is called as deciphering.

\section{Proposed System}

In this section we described the proposed transmitter and receiver section of the system
1) Transmitter section of the system consist of the microcontroller(STM32F401RE) nucleo board, Zig-bee module ,GLCD and power supply unit; In this section the data is encrypted and displayed in GLCD and then transmitted through the Zig-bee

2) Receiver section of the system consist of the microcontroller (STM32F401RE) nucleo board, Zig-bee module, Keypad, GLCD and Power supply unit; In this section the data is received by the Zig-bee and then decrypted, after entering the password the data is displayed on GLCD

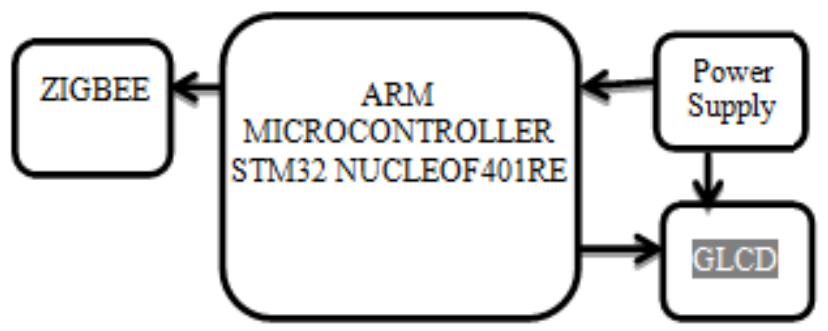

[a] Transmitter Section

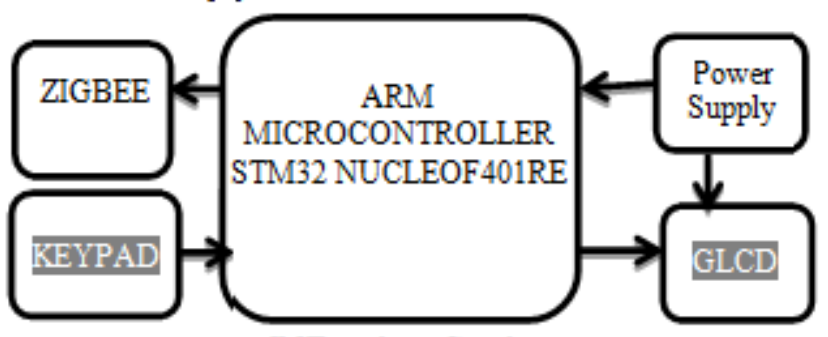

[b]Receiver Section

Figure 1: Block Diagram

\section{Process Flow}

1) Initialize the system first by fetching data which is being displayed on GLCD. This include the transmitter section

2) Then the xbee modules will be initialized which transmits from one X-bee module to another

3) The initial data will be displayed on GLCD through receiver X-bee module 


\section{International Journal of Science and Research (IJSR) \\ ISSN (Online): 2319-7064 \\ Index Copernicus Value (2015): 78.96 | Impact Factor (2015): 6.391}

4) The data will be decoded only via. Entering the password through keypad. If the correct password is entered then only the data will be decoded

5) The decoded data will be displayed on GLCD

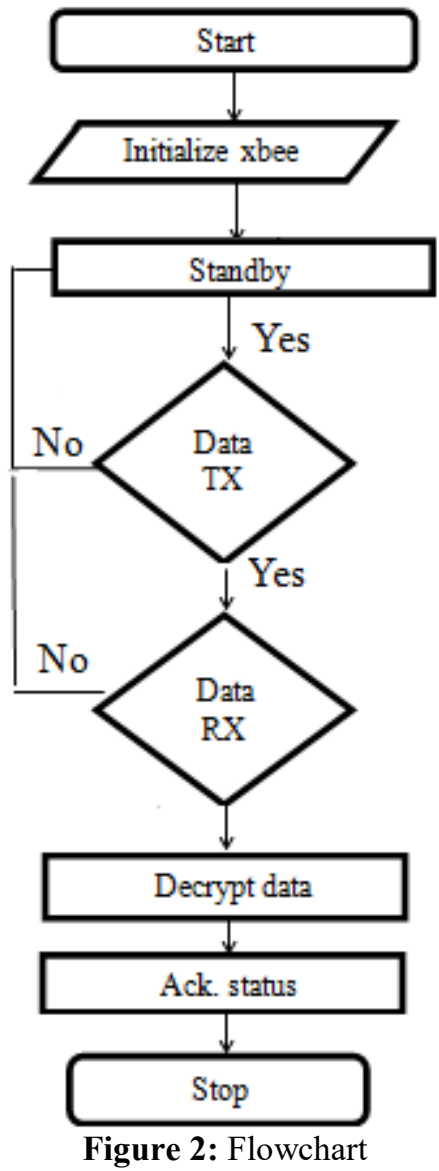

\section{Related Work}

[1]In this paper they proposed a high level evolution of Mobile Wireless Communication Networks from $1 \mathrm{G}$ to $4 \mathrm{G}$. An implementation of security algorithm is shown. This shows the way of securely text message transmission over wireless communication channel. For security a message is encrypted using Play fair encryption algorithm and then sent through wireless communication channel. At the receiver end the message is decrypted and the original message retrieved. First the original key message is encrypted with the key it is decrypted at the receiver end by entering the key

[2] In this research paper the author proposed; The use of Wireless sensor network requires the Zig-bee(XBEE) and IEEE 802.15.4 standard-based protocol which defines the physical, MAC layer, network layer and application layers. For sensor network applications, In this paper the conceptual study of IEEE 802.15.4 standard and Zig-bee based wireless sensor isdiscussed. This paper presents the overview of Zigbee protocol in terms of network topologies, architecture in terms of protocol. The protocol stack and its layers fundamental is also given briefly. The study and working of the network components is explained.

[3]This paper represents an image transmission system by using wireless sensor networks likeZig-bee and GPRS network. The Zig-bee wireless network uses mesh network topology, which is easy to connect through the image transmission system, it can structure the transmission data single by WSNs. It will have a fine application outlook in the image monitor system.

[4]In their research paper "WIRELESS DATA TRANSFER" they proposed the study of the Zig-bee the displayed the data on the notice board which is transmitted and received by the help of Zig-bee transmitter and receiver. As the communication is through Zig-bee (Xbee) the range of communication is around $300-400 \mathrm{~m}$.Zig-bee is worthwhile since it saves paper, constructing it more ecofriendly.

[5]The author proposed the security mechanism for the wireless data transfer by the combination of both cryptography and steganography

It services cryptographic algorithm self-possessed with steganography. In this paper, the filter bank cipher is used to encrypt the top-secret text message, it afford high level of safekeeping, scalability and speed. After that, a discrete wavelet transforms (DWT) established steganography is engaged to pelt the encrypted message in the cover image by transforming the wavelet factors. The demonstration of the acclaimed system is using peak signal to noise ratio (PSNR) andhistogram exploration

\section{Result}
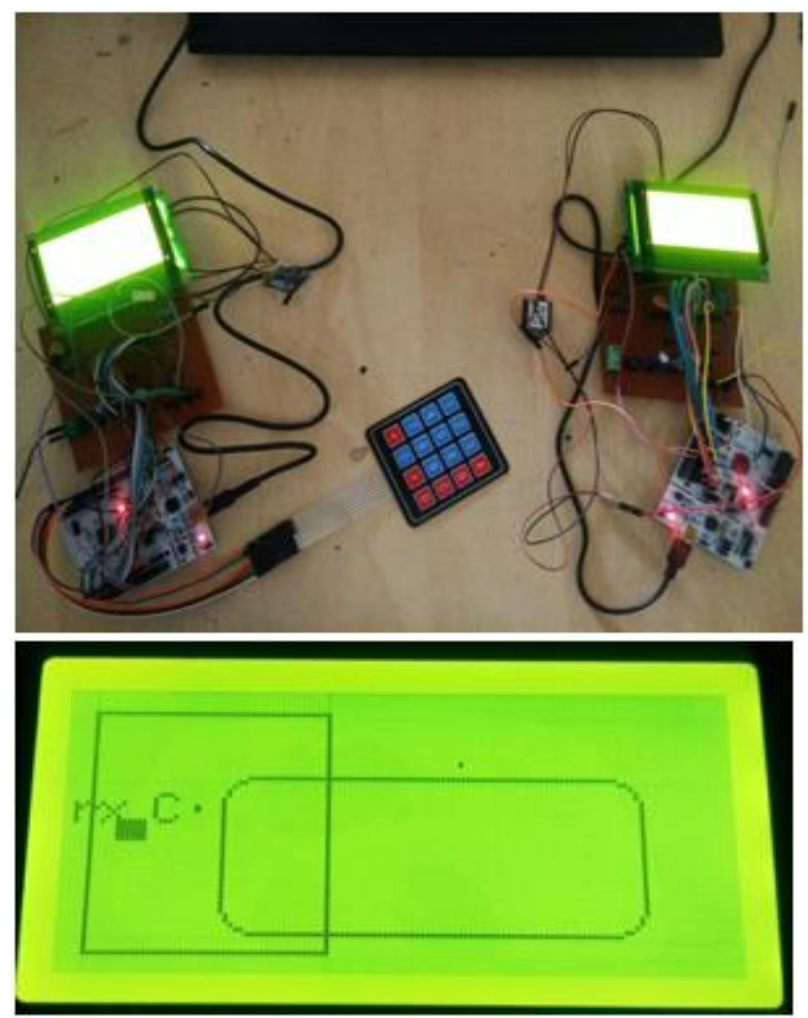


\section{International Journal of Science and Research (IJSR) \\ ISSN (Online): 2319-7064}

Index Copernicus Value (2015): 78.96 | Impact Factor (2015): 6.391

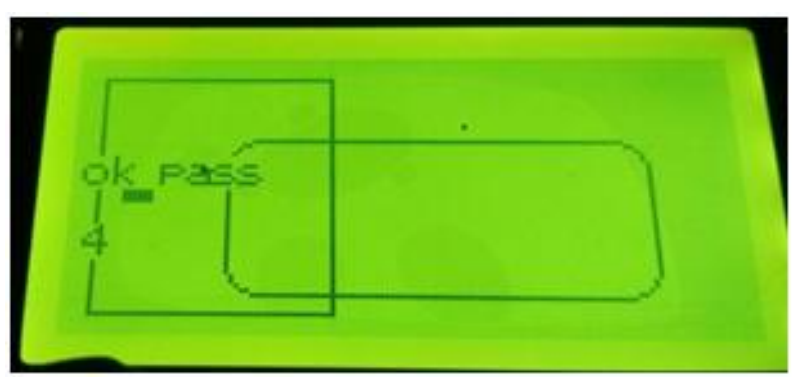

\section{Conclusion}

Wireless communication is an easy way to provide communication at places where there is no wired infrastructure. In this project, the Zig-bee provide low power consumption, low cost and simple wireless and secure communication encryption and decryption of the data is done. This system is small, simple, cost effective and good for wireless secure communication.

\section{Future Scope}

Zig-bee has very promising future in front of it. Zig-bee would provide revolutionizing statistics in upcoming years which would change the wireless world. This project can also be used in consumer, commercial and government markets. Due to data security and integrity this can be used in military applications. Furthermore advancement is possible by the combination of two or more cryptography techniques so therefore security of the data can be increased also using the Zig-bee in mesh network we can increase the range of communication

\section{References}

[1] Secure message transmission over wireless communicationMd. MizanurRahman and Farhan.Dept. of ICE, Rajshahi University, Rajshahi, Bangladesh Corresponding author Research Journal of Physical and Applied Sciences 2013 WudpeckerJournalsVol. 2(3), pp. 030 - 035, June 2013

[2] IEEE 802.15.4 and Zig-bee: A Conceptual Study Ms.Dharmistha D. Vishwakarma International Journal of Advanced Research in Computer and Communication Engineering Vol. 1, Issue 7, September 2012

[3] Xu Liu, Da Zhang, Xiao Lv, and Feng Jin Research of Image Transmission System Based on Zig-bee and GPRS NetworkInternational Journal of Machine Learning and Computing, Vol. 3, No. 5, October 2013

[4] Image Transmission over Noisy Wireless Channels Using HQAM and Median Filter International Journal of Information and Electronics Engineering, Vol. 3, No. 5, September 2013

[5] Wireless Data Transfer ZarnaPareks,HansalShahShivanBhatacharjee International journal of computer Technology VOL .5 NO.3 September 2013

[6] "Secure data communication system using cryptography and steganography" by Salah Saraieh International Journal of Computer Networks \& Communications (IJCNC)Vol.5, No.3, May 2013
[7] An Overview of Cryptographic Hash Functions and its uses of SANS Institute

[8] Zig-bee Wireless sensor Networks and their Applications Meng-Shiuan Pan and Yu- Chee Tseng Department of Computer Science National Chiao Tung University Hsin-CHU,30010,Taiwan

[9] The Role Of Zig-bee Technology In Future Data Communication System DR. S.S Riaz Ahmed Professor \&Head.Dept of Computer Applications,MohamedSathakEng. college Kilakarai\&PrincipalSathak Institute Of Technology Tamil Nadu India 623501

[10] Secure message transmission through Ad-Hoc network through dynamic key cryptography and comparative study with intrusion detection system by Navneet singh sikarwar in International journal of Emerging technology and advanced engineering

[11]BL-MLE: Block level message locked encryption for secure large file deduplication by Rongmao Chen, Yi $\mathrm{Mu}$, and Guomin Yang and FuchunGuo IEEE transactions oninformation forensics and security Vol.10 No.12 December 2015 